\title{
Development of a colorimetric assay for rapid quantitative measurement of clavulanic acid in microbial samples
}

\author{
DAI XiDa ${ }^{1 \dagger}$, XIANG SiHai $^{2 \dagger}$, LI Jia $^{2}$, GAO Qiang ${ }^{1 *} \&$ YANG KeQian ${ }^{2 *}$ \\ ${ }^{1}$ Key Laboratory of Industrial Fermentation Microbiology, Ministry of Education, College of Biotechnology, \\ Tianjin University of Science and Technology, Tianjin 300457, China; \\ ${ }^{2}$ State Key Laboratory of Microbial Resources, Institute of Microbiology, Chinese Academy of Sciences, Beijing 100101, China
}

Received December 14, 2011; accepted January 17, 2012

\begin{abstract}
We developed a colorimetric assay to quantify clavulanic acid (CA) in culture broth of Streptomyces clavuligerus, to facilitate screening of a large number of $S$. clavuligerus mutants. The assay is based on a $\beta$-lactamase-catalyzed reaction, in which the yellow substrate nitrocefin $\left(\lambda_{\max }=390 \mathrm{~nm}\right)$ is converted to a red product $\left(\lambda_{\max }=486 \mathrm{~nm}\right)$. Since CA can irreversibly inhibit $\beta$-lactamase activity, the level of CA in a sample can be measured as a function of the $A_{390} / A_{486}$ ratio in the assay mixture. The sensitivity and detection window of the assay were determined to be $50 \mu \mathrm{g} \mathrm{L}^{-1}$ and $50 \mu \mathrm{g} \mathrm{L}^{-1}$ to $10 \mathrm{mg} \mathrm{L}^{-1}$, respectively. The reliability of the assay was confirmed by comparing assay results with those obtained by HPLC. The assay was used to screen a pool of 65 S. clavuligerus mutants and was reliable for identifying CA over-producing mutants. Therefore, the assay saves time and labor in large-scale mutant screening and evaluation tasks. The detection window and the reliability of this assay are markedly better than those of previously reported CA assays. This assay method is suitable for high throughput screening of microbial samples and allows direct visual observation of CA levels on agar plates.
\end{abstract}

clavulanic acid, Streptomyces clavuligerus, nitrocefin, colorimetric assay, $\beta$-lactamase

Citation: Dai X D, Xiang S H, Li J, et al. Development of a colorimetric assay for rapid quantitative measurement of clavulanic acid in microbial samples. Sci China Life Sci, 2012, 55: 158-163, doi: 10.1007/s11427-012-4287-x

The fermentation titer of a metabolite is a key factor for its successful production. A convenient assay is required to evaluate the levels of a metabolite or product produced by various mutants, so that high-producing strain(s) can be rapidly identified. Clavulanic acid (CA) is a $\beta$-lactam antibiotic produced by Streptomyces clavuligerus. It is clinically used as a wide-spectrum irreversible $\beta$-lactamase inhibitor to treat various types of infections [1]. We recently reported a double-reporter guided mutant selection (dRGMS) method to generate CA over-producing mutants of $S$. clavuligerus [2]. To screen CA titers in the mutant pool, a convenient quantitative assay for CA is required. Previously, an HPLC method was developed as the gold standard for

†Contributed equally to this work

*Corresponding author (email: qgaoamtf@gmail.com; yangkq@im.ac.cn) quantitative measurement of $\mathrm{CA}$, for which $\mathrm{CA}$ must be imidazole-derivatized [3]. A bioassay-HPLC method based on $\beta$-lactamase-catalyzed hydrolysis of penicillin $\mathrm{G}$ was also developed to measure the levels of CA in culture broth of S. clavuligerus [4,5]. In that method, residual penicillin G is detected by a bioassay or by HPLC. Both methods are difficult to automate. Another improved $\beta$-lactamase inhibition-based method for CA detection [6] involved the use of a chromogenic substrate to replace the colorless penicillin $\mathrm{G}$. That method has been developed for applications using clinical samples [7-10].

Here, we report a similar colorimetric, $\beta$-lactamase inhibition-based assay specifically developed to detect CA in $S$. clavuligerus culture broth. In the assay, the chromogenic substrate nitrocefin (yellow $\lambda_{\max }=390 \mathrm{~nm}$ ) is hydrolyzed to 
form a red product $\left(\lambda_{\max }=486 \mathrm{~nm}\right)$ by $\beta$-lactamase. The absorption of both substrate $\left(A_{390}\right)$ and product $\left(A_{486}\right)$ is measured, and the $A_{390} / A_{486}$ ratio is used to calculate the CA concentration. These improvements greatly increase the CA detection window, compared with those of previously reported assays. The assay can be readily adapted for use in a microplate format to screen a large number of samples.

\section{Materials and methods}

\subsection{Bacterial strains and plasmids}

We used the wild-type strain S. clavuligerus NRRL 3585 and a strain transformed with the reporter vector pDRccaR, designated as $S$. clavuligerus pDRccaR [2]. M1 to M65 were 65 dRGMS mutants of $S$. clavuligerus pDRccaR selected for strong expression of the reporter. In the reporter construct, the over-expression target was $c c a R$, the pathway-specific transcriptional activator gene in CA biosynthesis [11].

\subsection{Culture conditions and selection of S. clavuligerus mutants by dRGMS}

The culture conditions and the dRGMS protocol were the same as those reported previously [2], but the dRGMS mutants used in this study were selected independently.

\subsection{Crude $\beta$-lactamase extract prepared for nitrocefin assay}

The source of the class A $\beta$-lactamase was a strain of Escherichia coli BL21(DE3) carrying the plasmid pET-23b(+) which contains a gene encoding $\beta$-lactamase. To prepare crude $\beta$-lactamase extract, the $E$. coli strain was cultured to $A_{600}=1.0$ in LB medium containing $50 \mathrm{mg} \mathrm{L}^{-1}$ ampicillin and then centrifuged to recover the cell pellet. The pellet was washed once with sodium phosphate buffer $(100 \mathrm{mmol}$ $\mathrm{L}^{-1}, \mathrm{pH}$ 7.0) and re-suspended in an equal volume of the same buffer. The cell suspension was sonicated on ice ( $4 \times 15 \mathrm{~s}$ at $30-\mathrm{s}$ intervals) and centrifuged to obtain the cell-free extract, which was used as the crude enzyme for the nitrocefin assay. The assay was performed in a 96-well plate (Costar). The specific activity of the enzyme extract was determined in a $100 \mu \mathrm{L}$ reaction mixture consisting of $100 \mathrm{mmol} \mathrm{L}^{-1}$ Na-phosphate buffer ( $\mathrm{pH}$ 7.0) containing 8 $\mu \mathrm{L}$ crude $\beta$-lactamase extract and $4 \mathrm{nmol}$ nitrocefin. The mixture was incubated at $30^{\circ} \mathrm{C}$. The amount of enzyme that hydrolyzes $1 \mathrm{nmol}$ nitrocefin in the first minute was defined as one unit of activity. The specific activity of the enzyme extract prepared in this study was $82 \mathrm{U} \mathrm{mL}^{-1}$ (see Appendix in the electronic version for details).

\subsection{Microplate assay for determination of $\mathrm{CA}$ concen- tration}

Nitrocefin (98\% purity) was dissolved in dimethylsulfoxide to a final concentration of $0.4 \mathrm{mmol} \mathrm{L}^{-1}$. The reactions were carried out in a 96-well plate. In each test well, $8 \mu \mathrm{L}$ crude $\beta$-lactamase extract and $10 \mu \mathrm{L}$ of a CA standard (Sigma-Aldrich) or a CA sample were mixed, and sodium phosphate buffer $\left(100 \mathrm{mmol} \mathrm{L}^{-1}, \mathrm{pH}\right.$ 7.0) was added to a final volume of $90 \mu \mathrm{L}$. We used $10 \mu \mathrm{L}$ sodium phosphate buffer as the blank. The mixture was incubated at $30^{\circ} \mathrm{C}$ for $20 \mathrm{~min}$ before addition of $10 \mu \mathrm{L}$ nitrocefin solution. The mixture was mixed well and then incubated at $30^{\circ} \mathrm{C}$ for $1 \mathrm{~h}$. The microplate was read at 390 and $486 \mathrm{~nm}$ to acquire $A_{390} / A_{486}$ ratios.

\subsection{Determination of CA concentration by HPLC}

The samples were analyzed by HPLC (Shimadzu LC-20AT) after derivatization by imidazole [3]. Briefly, the imidazole-derived CA-containing samples were loaded onto a reverse-phase column (Dikma Diamonsil-C18, $5 \mu \mathrm{m}, 250$ $\mathrm{mm} \times 4.6 \mathrm{~mm}$ ) and detected at $317 \mathrm{~nm}$. The mobile phase was an isocratic buffer system consisting of $\mathrm{NaH}_{2} \mathrm{PO}_{4}(0.1$ mol L ${ }^{-1}$, pH 3.68, adjusted with acetic acid) plus $6 \%$ methanol, and the flow rate was $1.0 \mathrm{~mL} \mathrm{~min}^{-1}$. Under these conditions, the retention time for the $\mathrm{CA}$ derivative was $15 \mathrm{~min}$.

\subsection{Nitrocefin disk assay for in situ detection of CA production in colonies on agar plates}

Strains were inoculated onto soybean agar plates and cultivated at $28^{\circ} \mathrm{C}$ for $3 \mathrm{~d}$ before detection of CA. A $10-\mu \mathrm{L}$ aliquot of $\beta$-lactamase solution was added to the center of a three-layer filter paper disc. The disk was air-dried, placed close to (but not touching) each tested colony, and incubated at $30^{\circ} \mathrm{C}$ for $30 \mathrm{~min}$ to allow $\mathrm{CA}$ in the agar to diffuse into the paper disk and inactivate the enzyme. Then, $10 \mu \mathrm{L}$ nitrocefin $\left(0.4 \mathrm{mmol} \mathrm{L}{ }^{-1}\right)$ was added to the paper disk and the plate was incubated at $30^{\circ} \mathrm{C}$ for another $20 \mathrm{~min}$. Colors of the disks were visually observed.

\section{Results}

\subsection{CA standard curve for microplate assay}

Aqueous solutions of CA show very low ultraviolet absorption at wavelengths above $230 \mathrm{~nm} \mathrm{[12].} \mathrm{Therefore,} \mathrm{the} \mathrm{CA}$ itself would not interfere with absorption measurements at $390 \mathrm{~nm}$ (nitrocefin) or $486 \mathrm{~nm}$ (hydrolyzed product). To establish the $\beta$-lactamase inhibition assay, CA standards of different concentrations were prepared and incubated with $\beta$-lactamase and nitrocefin. PBS buffer with no CA was 
used as the control. The sensitivity of the assay was determined by Student's $t$-test (Table 1). When a single wavelength was used to measure CA concentration, the lower detection limit was $0.1 \mathrm{mg} \mathrm{L}^{-1}$ for $A_{390}$ and $0.5 \mathrm{mg} \mathrm{L}^{-1}$ for $A_{486}$. However, when the ratio of $A_{390} / A_{486}$ was used instead, the lower detection limit was $50 \mu \mathrm{g} \mathrm{L}^{-1}$. We also analyzed the detection window of the assay; that is, the range of CA concentrations that give a good linear correlation with light absorbance. The detection window was from 0.1 to $2.0 \mathrm{mg}$ $\mathrm{L}^{-1}$ when $A_{390}$ was used and from 0.5 to $2.0 \mathrm{mg} \mathrm{L}^{-1}$ for $A_{486}$ (Figure 1A). At concentrations above $4.0 \mathrm{mg} \mathrm{L}^{-1}$, a significant increase in the CA concentration only resulted in a very small change in $A_{390}$ or $A_{486}$, thus reducing the resolution of the assay. When the ratio of $A_{390} / A_{486}$ was used instead (Figure 1B), the detection window was expanded from 50 $\mu \mathrm{g} \mathrm{L}^{-1}$ to $10 \mathrm{mg} \mathrm{L}^{-1}$, which is at least ten fold broader than the detection windows using $A_{390}$ or $A_{486}$. The linear function fitted from this linear region was as follows:
$\left(A_{390} / A_{486}\right)=0.334 \times \mathrm{CA}$ concentration+0.279 (Figure 1C). Both the slope $(0.334)$ and correlation coefficient $\left(R^{2}, 0.987\right)$ are sufficiently accurate to construct a calibration curve. Therefore, the ratio of $A_{390} / A_{486}$ was chosen as the independent variable to measure $\mathrm{CA}$ concentration. A CA standard curve was prepared for each microplate to eliminate possible plate-to-plate variations.

\subsection{CA over-producing mutant screening by the mi- croplate assay}

We selected 65 dRGMS mutants by the standard dRGMS method [2] and their CA titers were determined by the nitrocefin assay and compared with that of the starting strain. To ensure that CA concentrations in most samples were within the detection window, cultures of all strains were diluted with 30-volumes of buffer. Because the CA titer of the starting strain peaks on the third day of culture (Figure 2A),

Table 1 Sensitivity of CA microtiter plate assay ${ }^{\text {a) }}$

\begin{tabular}{|c|c|c|c|c|c|c|c|c|c|c|c|c|}
\hline $\begin{array}{l}\text { CA concentration } \\
\left(\mathrm{mg} \mathrm{L}^{-1}\right)\end{array}$ & & $A_{390}$ & & & $A_{486}$ & & & $A_{390} / A_{48}$ & & $\begin{array}{c}P \text {-value } \\
\left(A_{390} / A_{486}\right)\end{array}$ & $\begin{array}{c}P \text {-value } \\
\left(A_{390}\right)\end{array}$ & $\begin{array}{c}P \text {-value } \\
\left(A_{486}\right)\end{array}$ \\
\hline 0 & 0.026 & 0.026 & 0.032 & 0.079 & 0.078 & 0.09 & 0.339 & 0.333 & 0.346 & - & - & - \\
\hline 0.01 & 0.0308 & 0.029 & 0.028 & 0.09 & 0.086 & 0.082 & 0.342 & 0.342 & 0.337 & 0.766 & 0.589 & 0.459 \\
\hline 0.05 & 0.032 & 0.035 & 0.031 & 0.086 & 0.087 & 0.082 & 0.353 & 0.347 & 0.356 & 0.048 & 0.116 & 0.554 \\
\hline 0.1 & 0.037 & 0.035 & 0.033 & 0.076 & 0.071 & 0.069 & 0.376 & 0.372 & 0.378 & 0.001 & 0.039 & 0.077 \\
\hline 0.5 & 0.039 & 0.035 & 0.034 & 0.064 & 0.073 & 0.067 & 0.744 & 0.586 & 0.620 & 0.003 & 0.034 & 0.037 \\
\hline
\end{tabular}

a) Each well contained a mixture of $8 \mu \mathrm{L} \beta$-lactamase extract, $10 \mu \mathrm{L} \mathrm{CA}$ standard sample (Sigma-Aldrich), and $72 \mu \mathrm{L}$ Na-phosphate buffer (100 mmol $\mathrm{L}^{-1}, \mathrm{pH}$ 7.0). Plates were incubated at $30^{\circ} \mathrm{C}$ for $20 \mathrm{~min}$; then $10 \mu \mathrm{L}$ nitrocefin solution was added, and the mixture was incubated at $30^{\circ} \mathrm{C}$ for $1 \mathrm{~h}$ before reading $A_{390} / A_{486}$. Each CA standard sample was measured in triplicate. Statistical differences in $A_{390} / A_{486}$ ratio between a CA concentration and negative control (distilled water) were tested by Student's $t$-test at $P=0.05$ ( $n=3$, two tailed). Significant $P$-values $(\leqslant 0.05)$ are shown in bold type.
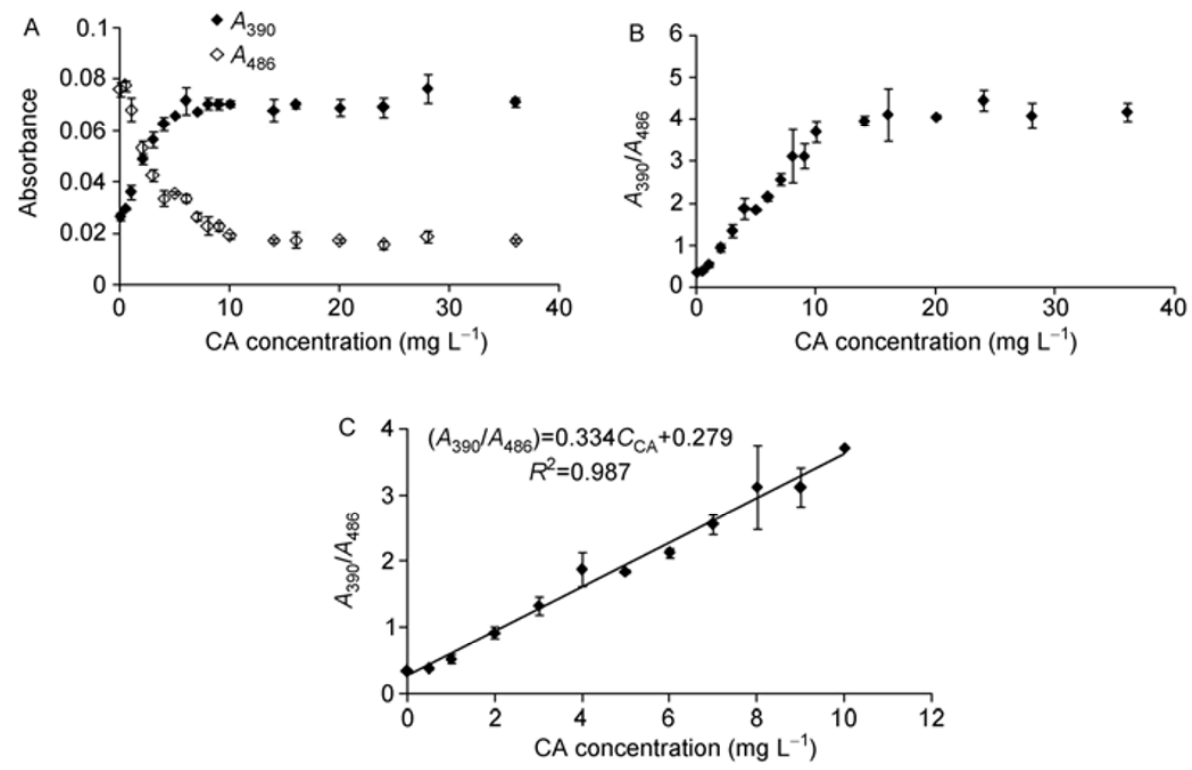

Figure 1 Standard curve for CA concentration calibration in microplate assay. A, CA concentration as a function of $A_{390}(\diamond)$ or $A_{486}(\diamond)$. B, CA concentration as a function of $A_{390} / A_{486}$ ratio. C, Detection window of assay when $A_{390} / A_{486}$ ratio was used to measure CA concentration. $\mathrm{C}$ is a highlight of B; $R^{2}$, correlation coefficient; $C_{\mathrm{CA}}$, CA concentration $\left(\mathrm{mg} \mathrm{L}^{-1}\right)$. Each concentration of $\mathrm{CA}$ was measured in triplicate. 


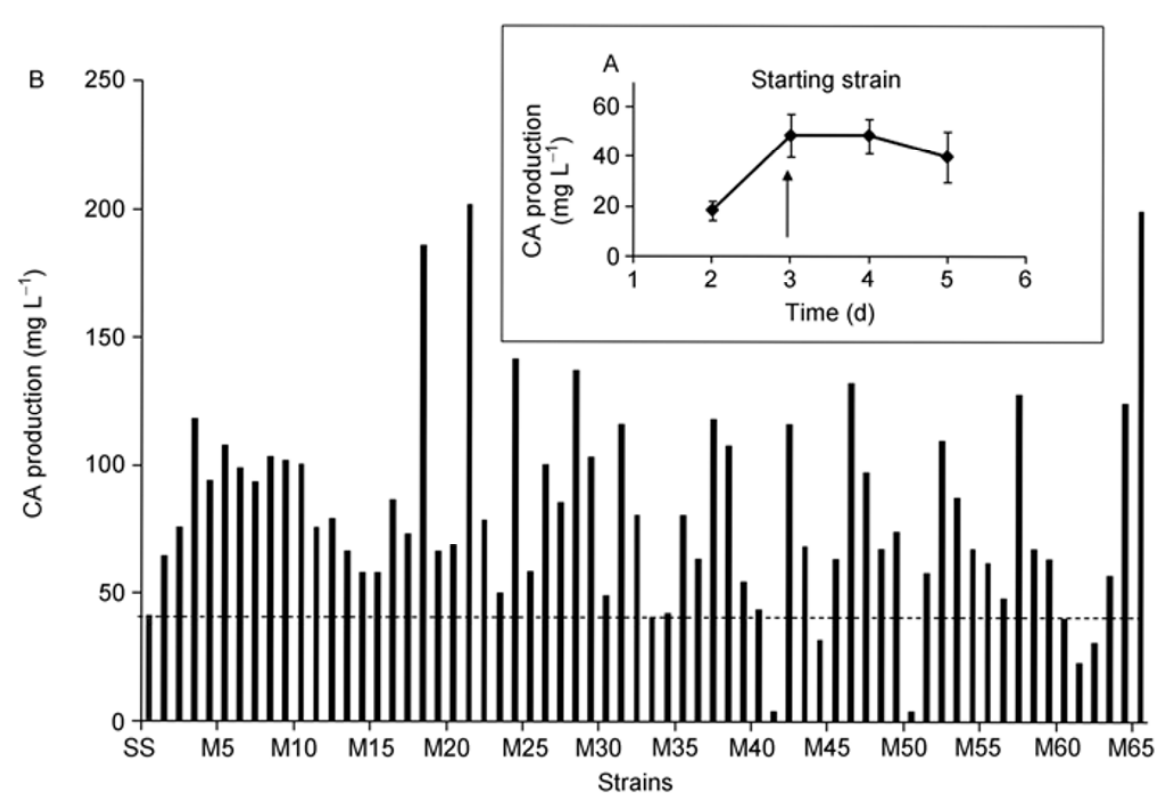

Figure 2 Evaluation of CA titers of 65 mutants by microplate assay. A, Time course of CA titers for starting strain. Arrow indicates when culture broth was collected. For each strain, cultures were evaluated in triplicate. B, CA titers of 65 mutants. SS, starting strain. Basic protocol was the same as that used for detection of CA standards except that cultures of all strains were diluted with 30-volumes Na-phosphate buffer before the assay. To increase the screening efficiency for initial screening to identify candidates, only one culture of each mutant was assayed (starting strain was evaluated using triplicate cultures).

the cultures of all mutants were collected at this time. As shown in Figure 2B, CA titers of most mutants were higher than that of the starting strain, similar to the previous report [2]. Three high-producing mutants, M18, M21, and M65, were identified as candidates and subjected to time-course investigation.

Before further evaluation of the mutants, we investigated whether other metabolites besides $\mathrm{CA}$ in the fermentation broth might interfere with the assay. Culture broths of the starting strain, the three high-producing mutants, and 10 randomly selected mutants were measured by HPLC. As shown in Figure 3, although it was not perfectly correlated $\left(R^{2}=0.948\right)$, there was a strong linear fit between the two

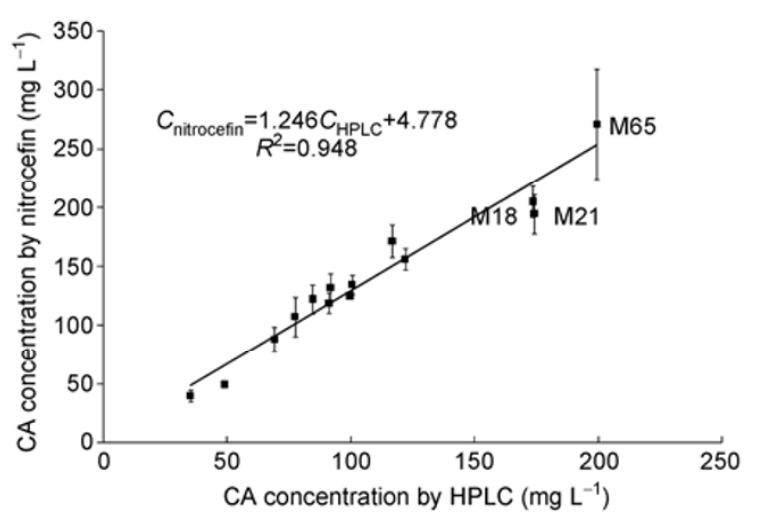

Figure 3 Correlation between CA concentrations determined by microplate assay $\left(C_{\text {nitrocefin }}\right)$ and HPLC $\left(C_{\text {HPLC }}\right) . R^{2}$, correlation coefficient. M18, M21, and M65, three CA-overproducing mutants. For each culture broth, HPLC test was conducted once and microplate test was conducted in triplicate. methods, indicating that the assay works well for $S$. clavuligerus cultures.

\subsection{Time-course evaluation of three high-producing mutants by the colorimetric assay}

Because the CA production level varies with culture time, we conducted a time-course evaluation of CA titers of the three high-producing mutants using the nitrocefin assay. As shown in Figure 4, the profiles of CA titer were dissimilar among the three mutants. M21 and M65 produced the highest CA titers on day 3, whereas M18 produced the highest level of CA on day 4. Compared with the other mutants, M18 showed a more stable CA titer. CA degradation is a well-known problem in fermentation [13,14], and the slow decline in the CA concentration in the culture of M18 is an

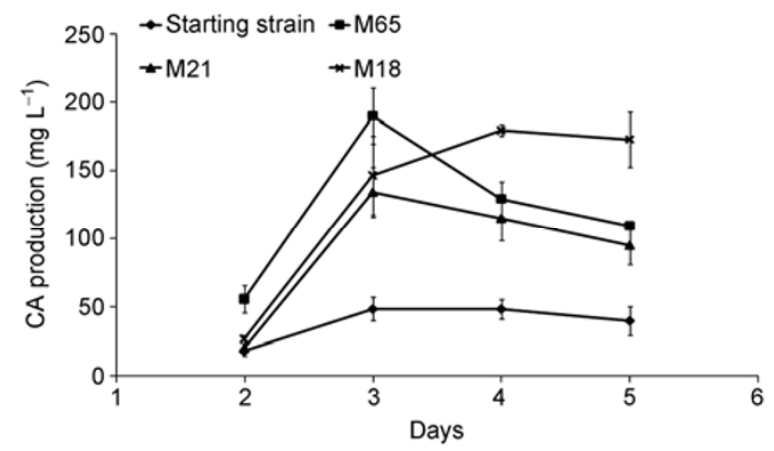

Figure 4 Time course measurements of CA titers of three over-producing mutants by microplate assay. Strains: starting strain $(\bullet)$, M65 (घ), M21 ( $\mathbf{\Delta})$, M18 (×). Triplicate cultures of each strain were evaluated. 
interesting phenotype worthy of further investigation. The levels of CA produced by these mutants were also measured by HPLC, and the results generally agreed with those obtained by the nitrocefin assay (data not shown).

\subsection{Visual detection of CA production levels by nitro- cefin assay}

An important advantage of a colorimetric method is that the color change can be directly observed. Visual detection is a useful alternative to instrument reading. As shown in Figure $5 \mathrm{~A}$, the solution in wells of the starting strain was reddish, while that in wells of over-producing mutants was bright yellow. We also developed a disk assay for rapid in situ detection of CA in colonies on an agar plate (see Materials and methods for details), as a complementary method to the microplate assay (Figure 5B and C). As shown in Figure 5B, the disk near the over-producing strain M65 was light yellow, while that near the starting strain was pink.

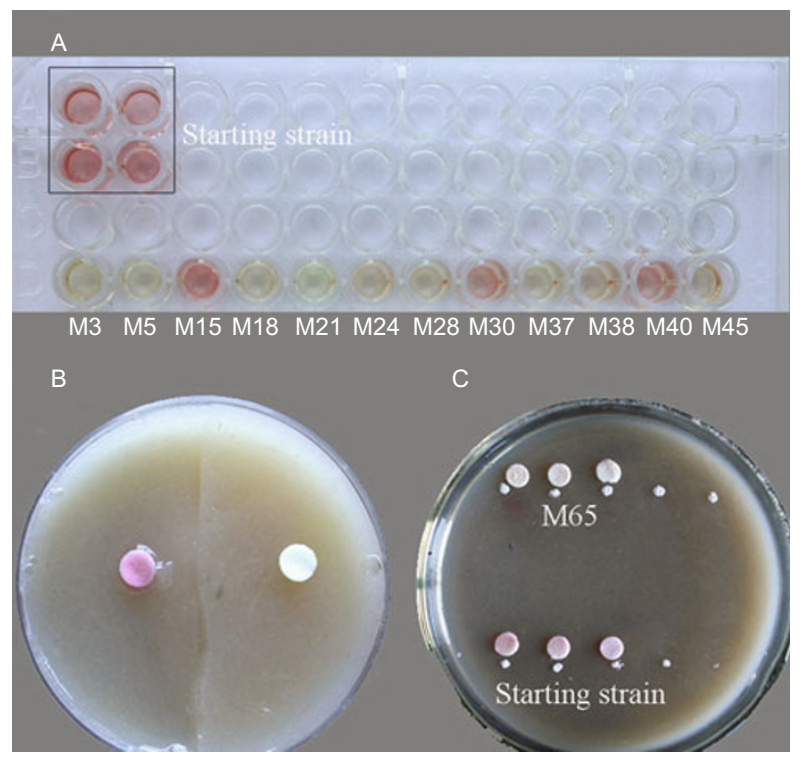

Figure 5 Visual detection of CA production levels by nitrocefin assay. A, Color differences among wells for various strains in microplate assay. Top four wells, starting strain; bottom 12 wells, mutants. B, Disk assay for in situ detection of CA concentration on hybrid agar plate: agar containing 20 $\mathrm{mg} \mathrm{L}^{-1} \mathrm{CA}$ (right), agar without CA (blank) (left). Paper disk pre-loaded with $\beta$-lactamase was on the left and right halves of the plate, and after 30 min incubation at $30^{\circ} \mathrm{C}$, nitrocefin was added to the disk to detect the remaining $\beta$-lactamase activity. C, Comparison between over-producing strain M65 (top five spots, three of which were tested by disk assay) and starting strain (bottom five spots, three of which were tested by disk assay).

\section{Discussion}

This is the first report of a colorimetric microplate assay for the quantitative measurement of CA in culture broth of $S$. clavuligerus. Compared with the previous bioassay-HPLC method, which is also based on $\beta$-lactamase inhibition but uses penicillin $\mathrm{G}$ as the substrate, our method offers obvious advantages. First, the reaction can be observed and measured as the color changes, and more importantly, our method can be easily automated. The assay could also be used for in situ visual detection of CA production.

Previously, a $\beta$-lactamase inhibition-based assay reported by Gutman et al. [4] had been used to detect CA in $S$. clavuligerus culture broth [5]. However, it was not successful because of interference caused by BLIP, a $\beta$-lactamase inhibitor protein produced by the host. In our study, we did not observe BLIP interference (Figure 3). If BLIP interference was a problem, the culture broths could be heated at $98^{\circ} \mathrm{C}$ for $2 \mathrm{~min}$ to inactivate BLIP before the assay, as this treatment does not affect the stability of $\mathrm{CA}$, as reported by Doran et al. [5].

For convenience, it is desirable that assays have a broad detection window. The $A_{390} / A_{486}$ ratio was used for the first time as an indicator of CA concentration, which made the detection window much broader than that of any $\beta$-lactamase inhibition assay reported previously $[4,6]$.

We thank Dr. Shahriar Mobashery (University of Notre Dame, IN, USA) for kindly providing the nitrocefin. This work was supported by the Young Scientists Fund (Grant No. 31000025) from the National Natural Science Foundation of China and National High Technology Research and Development Program of China (Grant No. 2012AA021302).

1 Ninane G, Joly J, Kraytman M, et al. Bronchopulmonary infection due to $\beta$-lactamase-producing Branhamella catarrhalis treated with amoxycillin/clavulanic-acid. Lancet, 1978, 2: 257

2 Xiang S H, Li J, Yin H, et al. Application of a double-reporter-guided mutant selection method to improve clavulanic acid production in Streptomyces clavuligerus. Metab Eng, 2009, 11: 310-318

3 Foulstone M, Reading C. Assay of amoxicillin and clavulanic acid, the components of Augmentin, in biological fluids with high-performance liquid chromatography. Antimicrob Agents Chemother, 1982, 22: 753-762

4 Gutman A L, Ribon V, Leblanc J P. Rapid and convenient spectrophotometric method for determination of clavulanic acid. Anal Chem, 1985, 57: 2344-2346

5 Doran J L, Leskiw B K, Aippersbach S, et al. Isolation and characterization of a beta-lactamase-inhibitory protein from Streptomyces clavuligerus and cloning and analysis of the corresponding gene. J Bacteriol, 1990, 172: 4909-4918

6 Legg B, Clarke J G, Davies B E, et al. Automated enzyme-inhibition assay for clavulanic acid in biological samples. J Pharm Biomed Anal, 1985, 3: 381-384

7 Mendiratta D K, Kaur H, Deotale V, et al. Status of high level aminoglycoside resistant Enterococcus faecium and Enterococcus faecalis in a rural hospital of central India. Indian J Med Microbiol, 2008, 26: 369-371

8 Tam V H, Schilling A N, Larocco M T, et al. Prevalence of AmpC over-expression in bloodstream isolates of Pseudomonas aeruginosa. Clin Microbiol Infect, 2007, 13: 413-418

9 Hedberg M, Lindqvist L, Tuner K, et al. Effect of clavulanic acid, sulbactam and tazobactam on three different $\beta$-lactamases from Bacteroides uniformis, Clostridium butyricum and Fusobacterium nucleatum. J Antimicrob Chemother, 1992, 30: 17-25

10 O'callaghan C H, Morris A, Kirby S M, et al. Novel method for detection of $\beta$-lactamases by using a chromogenic cephalosporin sub- 
strate. Antimicrob Agents Chemother, 1972, 1: 283-288

11 Santamarta I, Rodriguez-Garcia A, Perez-Redondo R, et al. CcaR is an autoregulatory protein that binds to the $c c a R$ and $c e f D-c m c I$ promoters of the cephamycin C-clavulanic acid cluster in Streptomyces clavuligerus. J Bacteriol, 2002, 184: 3106-3113

12 Fleming I D, Noble D, Noble H M, et al. Pure potassium salt of clavulanic acid. US Patent, 4367175, 1983-01-04
13 Brethauer S, Held M, Panke S. High concentrations of clavulanic acid but not of its degradation products decrease glycerol consumption and oxygen uptake rates in cultures of Streptomyces clavuligerus. Biotechnol Bioeng, 2008, 100: 439-447

14 Stirling I, Elson S W. Studies on the biosynthesis of clavulanic acid. II. Chemical degradations of ${ }^{14} \mathrm{C}$-labelled clavulanic acid. J Antibiot (Tokyo), 1979, 32: 1125-1129

Open Access This article is distributed under the terms of the Creative Commons Attribution License which permits any use, distribution, and reproduction in any medium, provided the original author(s) and source are credited. 